\title{
11 Fazit und Nachwort
}

\subsection{Fazit}

Ohne eine physikalische Interaktion keine Wahrnehmung! Der Mensch, genauso wie jedes andere Lebewesen, steht ausschließlich mittels EM-Wellen mit seinem Umfeld in Kontakt (sehen, fühlen, riechen, schmecken, hören); EM-Wellen, die als Produkt unterschiedlichster Quantenaktivitäten zu interpretieren sind. Als Konsequenz ergibt sich aus solchen Kontakten eine quantenphysikalische Verschränkung, die intensiviert, aber auch reduziert werden kann; wie gezeigt werden konnte eine Verschränkung mit weitreichenden Folgen, denn sie liefert den Zugang zu beliebigen Informationen auch nichtlokal, weil sämtliche Informationen auf elementarer Ebene codiert sind. Allein diese abstrakt vorliegenden Informationen benötigen noch eine Interpretation, um für das jeweilige lebende System eine Bedeutung zu bekommen.

Die derzeitigen Forschungen zeigen darüber hinaus, dass unser gesamter Wahrnehmungs- und Verarbeitungsapparat letztlich auf Basis von EM-/Quantenprozessen arbeitet und nur vor diesem Hintergrund vollständig zu verstehen ist. Aus diesem Grund muss der Mensch informationstheoretisch als ,Homo Physicus' und damit als Mixed-Zustand von Quanten- und klassischer Welt interpretiert werden, für den beide Welten Wirkung und damit Bedeutung haben. In ihm ist das Zusammenspiel zwischen physikalischen Ausgangsbedingungen und psycho-sozialen sowie ökonomischen Einflussprozessen repräsentiert. Dieses Zusammenspiel läuft in den Prozessen des Gehirns in der Ausprägung von ,Schrödinger's Katze' ab und lässt sich nicht direkt beobachten. Die Überlagerung unzähliger möglicher Informationen im Gehirn und deren Zusammenspiel bleiben verborgen bzw. unbestimmt. Nur das Ergebnis am Ende zeigt sich in unserer Wahrnehmung in Form eines Gedankens oder Bildes. Alles davor verschwindet in einen für uns nicht mehr erreichbaren Zustand.

Dieser ,Homo Physicus' und ,Schrödinger's Katze im Kopf' haben Konsequenzen für fast alle Bereiche unseres Lebens: Gesellschaft und Unternehmen, Management, Führung und Entscheidungen, Kommunikation, Lehre und Lernen, Psychologie, Medizin und Neurowissenschaften. Nicht zuletzt ergeben sich für die Naturwissenschaft selbst Konsequenzen, wenn wir uns diesen nicht nur erkenntnistheoretisch, sondern auch ökonomisch annähern. Die ökonomische Seite ergibt sich aus den finanziellen Mitteln, die für ihre Projekte gewährt werden und abhängig vom jeweiligen Paradigma sind. Verändert sich das wissenschaftliche Paradigma und in dessen Folge die Forschungsthemen, wie es derzeit beim Switch in Richtung Digitalisierung und Informationstechnologie zu beobachten ist, so ändert sich auch der Mittelfluss und in dessen Folge die Attraktivität der Themen.

Vor diesem Hintergrund wurde deutlich, dass Intuition als Ergebnis eines Zugriffs unseres körperlichen und mentalen Systems auf sämtliche, in der Welt existierende Informationen zu interpretieren ist und tatsächlich auch innerhalb bestimmter Grenzen bewusst gesteuert werden kann. SyA arbeitet mit dieser Intuition, indem eine Frage und 
damit eine bewusste Intention gesetzt werden und die Repräsentanten auf ihre darauffolgenden körperlichen und mentalen Wahrnehmungen achten. Dieser Prozess darf als direkte Analogie zu einem Rechenprozess in einem Quantencomputer angesehen werden, dem eine Rechenaufgabe eingegeben wird. Auch dort weiß man nicht, wie der genaue Weg zum Ergebnis verläuft, nur dass ein solches Ergebnis am Ende abgelesen werden kann. Wesentlich sind bei solchen Berechnungen mithilfe von Quantenrechnern der Einsatz von Fehlerkorrekturverfahren, nicht anders wie im täglichen Leben.

Bei beiden, SyA und Rechenprozess, kommt es letztlich auf die Auswahl und Qualität der Frage und schließlich auf die Interpretation der Ergebnisse an. In beiden Aspekten finden sich die zentralen Herausforderungen und auch Gefahren. Dieser Umstand unterscheidet sich allerdings in keiner Weise von sogenannten rationalen Vorgehensmodellen, weshalb sich daraus kein Vor- bzw. Nachteil für intuitive Verfahren ableiten lässt, wohl aber in der Handhabung der Ergebnisse berücksichtigt werden sollte.

Aus den derzeit existierenden Forschungen wurde eindringlich die bessere Qualität unbewusster, intuitiver Entscheidungen in komplexen Situationen deutlich; komplexe Situationen, wie sie die VUCA-Rahmenbedingungen erzeugen und in denen so gut wie nie alle Informationen, die für eine gute Entscheidung nötig wären, zur Verfügung stehen. Es wurde auch deutlich, dass erfolgreiche Entscheider vorzugsweise auf ihre intuitiven Eingebungen zurückgreifen und sich von ihnen leiten lassen. Erfolgreiche Unternehmensführung und strategisches Management sind ganz essenziell von solch intuitiven Prozessen abhängig, weshalb SyA nun als erklärbare Methode eine größere Chance für ihren Einsatz bekommen könnte. Die bisherige Ablehnung solcher Art gewonnener Erkenntnisse beruhte ausschließlich auf einer nicht vorhandenen Theorie und sollte deshalb in Zukunft wegfallen.

Abschließend darf noch einmal darauf verwiesen werden, dass die Grundlage für einen jetzt erklärbaren Prozess zur Wirkungsweise von Intuition und SyA vor allem in der Falsifikation weit verbreiteter Annahmen zu finden ist. Es konnten zahllose gleiche Phänomene in unterschiedlichen Kontexten gezeigt und damit die Fehlannahmen von Anomalien oder unzureichender Messanordnungen widerlegt werden. In gleicher Weise gelang der Ausschluss klassischer Erklärungen, die einfach nicht für sämtliche Phänomene passen wollten. Weiter gelang die Überwindung von angenommenen Begrenzungen aus dem Dekohärenzmodell von Zeh mithilfe der Falsifizierung des Tieftemperatur- und Makrosystem-Paradigmas, das relevante quantenphysikalische Prozesse bei normalen Umweltbedingungen in lebenden Systemen und im Gehirn verneinte.

Unterstützt wurden diese Falsifikationen durch eine gleichzeitige Bestätigung der existierenden Theorien zu einem möglichen Quantenverhalten in Makrosystemen, durch die gleichen Nachweisverfahren von Nicht-Lokalität in Physik wie bei lebenden Systemen und damit die Möglichkeit einer Quanten-Teleportation mit Verteilung von Information. Schließlich konnten auch quantenphysikalische Prozesse im Kontext neurowissenschaftlicher Aktivitäten gezeigt bzw. modelliert werden. Schließlich führte die Entdeckung homologer Zusammenhänge zwischen Quantenphysik, Systemtheorie und SyA zu einer gemeinsamen Wurzel, womit die bisherigen Rückgriffe auf analoge bzw. metaphorische Erklärungen obsolet wurden. 
Vervollständigt wurden die Modelle durch eine Normierung des Informationsbegriffs über verschiedene Wissenschaftsdisziplinen hinweg sowie die Idee einer Normierung der Psi-Funktion $(\Psi)$, einen Beitrag zur Theory of Mind und eine Idee, wie Quantenfeldtheorie, Allgemeine Relativitätstheorie und Quantengravitation zusammenspielen könnten.

\subsection{Nachwort}

Diese Forschung entwickelte sich aus der Neugierde heraus, das Besondere der SyA und ihrer innewohnenden Intuition zu verstehen. Als Ergebnis steht am Ende aber eher die Umkehrung dieses Bestrebens, nämlich die Ableitung eines allgemeinen Prozesses (der Informationswahrnehmung) aus dem Besonderen (der SyA) heraus. Ob diese Ergebnisse breite Akzeptanz finden oder ob sie das Schicksal des Vergessens bzw. Widerspruchs erleiden werden, entsprechend einer Beobachtung von Nisbett und Borgida bei Studenten, weil sie zu überraschend sind, wird sich zeigen: „,Der Unwille der Probanden, das Besondere aus dem Allgemeinen abzuleiten, reichte allenfalls an die Bereitwilligkeit heran, mit der sie aus dem Besonderen das Allgemeine folgerten " (Nisbett und Borgida in Kahneman 2016: 217).

Eine wesentliche Voraussetzung für Akzeptanz und Anwendung der hier vorgestellten Ergebnisse ist natürlich, dass die Schlussfolgerungen keiner Verzerrung aufgrund einer „Illusion der Gültigkeit“ (Kahneman 2016: 259-263) oder einem WYSIATI ${ }^{246}$-Effekt (ebd. 112-115) unterliegen, wie sie Kahneman so schön herausgearbeitet hat. Die Hoffnung auf eine erfolgreiche Vermeidung von Verzerrungsfehlern resultiert aus der Vielzahl unterschiedlicher Beispiele aus unterschiedlichsten Disziplinen, in denen immer wieder die gleichen merkwürdigen und bis heute nicht erklärbaren Phänomene beobachtbar sind, womit Kahneman's Forderung nach Vielfältigkeit des Zugangs erfüllt sein sollte.

Die Akzeptanz wird aber auch davon abhängen, inwieweit die Forschungsgemeinde es schafft eine psychologisch als auch systemtheoretisch begründbare ,, theorieinduzierte Blindheit “ zu überwinden. Eine Blindheit, die mit der Akzeptanz und der Nutzung einer Theorie einhergeht, wie es Kahneman formuliert. Dies geschieht mit der Folge, ihre Schwächen nicht mehr bemerken zu können und sich ,,im Zweifelsfall zugunsten der Theorie und der Gemeinschaft der Experten, die sie für richtig hält, zu entscheiden “ (Kahneman 2016: 340).

Noch 1970 wurde es als exotisch angesehen, dass Quantenverschränkungen in chemischen Reaktionen eine Rolle spielen würden (Al-Khalili und McFadden 2015) und 2012 passten die Teilnehmer eines internationalen Workshops über Quantenbiologie noch in einen kleinen Hörsaal (ebd. 27). Anwesend waren die meisten Wissenschaftler, die sich mit diesem Thema befassten. Selbstverständlich waren all diejenigen nicht dabei, die zwar das gleiche Thema bearbeiteten, aber den sogenannten Parawissenschaften

${ }^{246}$ WYSIATI bedeutet: What you see is all there is. 
zugerechnet wurden, wie beispielsweise Sheldrake und sogenannte Quantenmystiker (vgl. ebd. 5). Ein erheblicher Teil der Personen und Gruppen, deren Erkenntnisse hier zusammengetragen und in ein übergreifendes Bild gestellt wurden, sind Außenseiter oder wurden mit Beginn ihrer Tätigkeit zu solchen gemacht. Dabei sind zunächst nicht erklärbare Rätsel (Beobachtungen und Erfahrungen) fast immer der Ausgangspunkt für ein neues Verständnis über unsere Welt. Ob Kopernikus (Sonne im Mittelpunkt), Max Planck (Portionierung der Energie) (ebd. 5) oder H.D. Zeh (Dekohärenztheorie), immer wurde an den Grenzen des aktuellen Verständnisses gearbeitet. Manchmal hatten die Forscher Glück und zeitnah Mitstreiter gefunden oder konnten ihre Erkenntnisse schnell und eindeutig nachweisen, oft wurden sie aber skeptisch betrachtet oder ausgegrenzt. Neue Entwicklungen und die Erkenntnisse der letzten paar Jahre geben Hoffnung, dass diese Arbeit einen Beitrag für ein verändertes Verständnis bei vielen Phänomenen leisten kann, für die es heute keine Erklärung und auch keine theoretischen Ansätze gibt.

Mit den hier vorgestellten Ergebnissen wird zudem auch klar, weshalb die Überprüfung dieser subtilen Phänomene so schwierig ist und sich nicht einfach wiederholen lässt und schon gleichgar nicht mit deterministischer Sicherheit. Die Quantenwelt ist einfach nicht deterministisch und lässt sich mit kleinsten Einflüssen zu völlig unterschiedlichen Resultaten animieren; Unterschiede, die in der rein physikalischen Forschung berechenbar sind, nicht aber in gleicher Weise in der Welt des Lebenden und nicht einfach Isolierbaren. Konsequenterweise müssen unsere bisherigen Studienannahmen und -designs auf den Prüfstand gestellt werden, mit der großen Wahrscheinlichkeit einer umfassenden Neuausrichtung.

In jedem Fall erscheint das bisher akzeptierte Verständnis von Feynman, dass niemand die Quantenphysik wirklich versteht, weil sie nicht Teil unser Alltagserfahrung sei (Wilde 2017: 24), nachhaltig infrage gestellt. Wir erleben die Quantenphysik tagtäglich in den verschiedensten Zusammenhängen. Dieser Umstand veranlasst mittlerweile auch Physiker dazu, Quantenphysik und die Bayessche Wahrscheinlichkeit zusammen zu denken (Fuchs u. a. 2014). Nur mit der Logik von Descartes im Kopf, der seine Wirkung über das Bayes-Theorem (Verzerrungseffekte) und den automatischen Auswahlprozessen auf neuronaler Ebene entfaltet, können sie von Vielen zwar wahrgenommen, von unseren Experten aber nicht akzeptiert werden (schwarzer Elefant). Die Folge ist ein Kampf zwischen Weltanschauungen und Paradigmen.

Deshalb lässt sich diese Forschung auch als Fortsetzung von Fechner's Überlegungen zu einer Psychophysik verstehen. Fechner versuchte einen komplementären Forschungsansatz zu entwickeln, indem er die Beziehung zwischen subjektiven (geistigen) Wahrnehmungen und objektiven (neuronalen oder körperlichen) Prozessen bzw. Ereignissen untersuchte. ,, The key idea underlying Fechner's psychophysics was that body and mind are just different reflections of the same reality " (Ehrenstein und Ehrenstein 1999: 1211). Es geht damit auch um die Suche nach einer Beziehung zwischen Geist und Materie und einer Auseinandersetzung mit dem cartesischen Denken und der Descartesschen Idee von der Trennung derselben, die Jahrhunderte besonders das west- 
liche Weltbild geprägt hat (Dijksterhuis und Roth 2010: 39; Damasio 2004; Whyte 1979).

Es mag schwierig sein, mit unserer jahrhundertalten Prägung basierend auf Descartes und Newton, die mit den hier entwickelten Überlegungen verbundenen Konsequenzen zu akzeptieren. Die abertausende von Experimenten aus verschiedensten Disziplinen, die dieser Forschungsarbeit zugrunde liegen und nicht von mir in eine gewünschte Richtung beeinflusst sind, sondern nur gesichtet und in einen thematischen Kontext gestellt wurden, lassen sich nicht so einfach wegdiskutieren. Diese Experimente werden heute auch von Kritikern als seriös und wissenschaftlich gut gemacht angesehen. Wenn sie dennoch infrage gestellt werden, kann dies entweder nur mit magischen, respektive dogmatischen oder andererseits mit metaphysischen Argumentationen vonstatten gehen. Metaphysisch versteht sich hier im Sinne der Philosophie, die versucht Fragen zu beantworten, die empirischen Experimenten nicht mehr zugänglich sind. Die allgemein gegen Forscher, die sich mit den vorgestellten Themen beschäftigen, verwendete und als abwertend gedachte Zuschreibung einer ,magischen' und ,nicht-fundierten' Denkweise (des Öfteren wird auch ,metaphysisch' mit dem Ziel der Abwertung verwendet) dreht sich nun offensichtlich und eindeutig um, was mit der folgenden letzten Abbildung (Abb. 101) veranschaulicht werden soll.

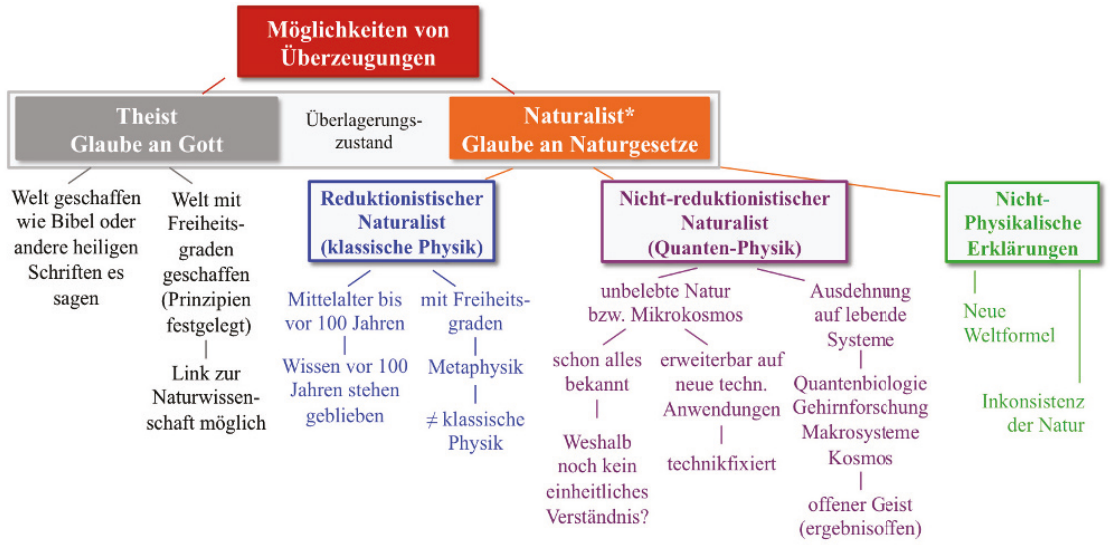

Abb. 101 | Möglichkeiten von Überzeugungen

(eigene Darstellung). In der Grafik sind unterschiedliche Überzeugungen dargestellt, die meist als Entweder-oder-Positionen verstanden werden. Bei genauer Betrachtung bieten sie allerdings auch einige Möglichkeiten von Superpositionen, unter denen scheinbar gegensätzliche Überzeugungen gleichwertig und gleichzeitig existieren können.

*Naturalist: alle Phänomene der Welt sind durch Naturgesetze bestimmt. Dazu gehören auch Phänomene wie Bewusstsein, Moral oder Ästhetik.

Dargestellt sind die Möglichkeiten von Überzeugungen zunächst in ihrer Grundpolarität: Glaube an Gott oder Glaube an Naturgesetze, die die Welt festlegen (Bergmann 
2019). Wie gleich deutlich werden wird, kann und will die hier vorgelegte Arbeit keine Entscheidungshilfe zwischen den beiden Möglichkeiten liefern. Ganz im Gegenteil, was sich abzeichnet ist eine Superposition beider Optionen. Je nach Perspektive und Fokussierung tritt entweder das eine oder das andere in den Vordergrund.

Die Optionen für Theisten lassen sich in zwei Überzeugungsrichtungen unterscheiden: (1) diejenigen, die an eine 1:1-Gültigkeit glauben, wie es in den jeweiligen heiligen Schriften beschrieben wurde und (2) diejenigen, die die Welt mit Freiheitsgraden ausgestattet sehen. Im ersten Fall produziert dies Gläubige à la Kreationisten und Islamisten mit hochgradig fundamentalistischen Ansichten. Im zweiten Fall wird Gott die Kompetenz zugeschrieben, entsprechende allgemeingültige Prinzipien festgelegt zu haben, aus denen heraus sich die Welt auf der Basis eigener Fähigkeiten weiterentwickelt. Diese letzte Überzeugung bietet einen tragbaren Link hinüber zu den Naturalisten und damit zu den Naturwissenschaften. Als Überlagerung zwischen diesen beiden theistischen Polen besteht jedoch auch die Option, dass Gott zusätzlich noch eingreifen kann.

Auf der Seite der Naturalisten bestehen drei Hauptunterscheidungen: Erstens, diejenigen, die nach wie vor an die klassische Physik glauben. Ihre Vorstellung ist entweder vor über 100 Jahren stehengeblieben und darf als, unwissend' tituliert werden, oder sie geben der klassischen Physik Freiheitsgrade, wodurch sie den physikalischen Rahmen verlassen und sich den ,metaphysischen' Erklärungen zuwenden.

Im Bereich der Quantenphysik wiederum glauben immer noch viele an die Beschränkung auf Mikrokosmos und unbelebte Natur. Manche, die zu dieser Überzeugung neigen, glauben, dass schon alles bekannt sei und tentieren zu einem gewissen Dogmatismus. Sie haben damit einen Weg der Nichtwissenschaftlichkeit eingeschlagen, den sie allerdings selbst nicht mehr bemerken. Hier stellt sich die Frage, weshalb noch kein einhelliges Verständnis erzielt werden konnte und zahlreiche Fragen tatsächlich noch offen sind. Andere wiederum haben die ursprünglichen Vorstellungen überwunden und beschäftigen sich mit viel Begeisterung mit den neuen Techniken wie Quantencomputern. Gleichzeitig verdrängt ein erheblicher Teil von ihnen die neuesten Entwicklungen im Bereich offener Quantensysteme oder bekommt sie einfach nicht mit. Sie glauben immer noch nicht daran, dass auch die belebte Natur Quantenprozesse realisiert. Die Technikfixierung neigt zu einer gewissen Verdrängung und Ignoranz. Die Gruppe, die sich auf offene Quantensysteme (Makrosysteme, Quantenbiologie, Neurowissenschaften und Kosmos) und damit auch auf lebende Systeme eingelassen hat, konnte sich offensichtlich einen offenen, neugierigen Wissenschaftsgeist bewahren. Sie neigen dazu, ergebnisoffen in ihre Forschung zu gehen und sich überraschen zu lassen. Vielleicht sind sie schlicht auch nur an anderen Themenkreisen interessiert oder haben eigene irritierende Erfahrungen gemacht, die nachhaltig wirken.

Schließlich lässt sich im Rahmen der Naturwissenschaften noch eine weitere Gruppe identifizieren, die nach einer neuen, vielleicht ganz anderen Weltformel suchet oder an die Inkonsistenz der Natur glaubt, wie bereits im Vorwort angemerkt. Für viele scheint dies etwas zu weit gesprungen und ist deshalb mit einem Hauch von Esoterik verbunden. Gleichwohl muss offenbleiben, ob sie nicht doch einen überraschenden, ganz neuen 
Zugang zum Verständnis der Welt offenlegen, denn der schwarze Schwan ist aus prinzipiellen Gründen nicht auszuschließen.

Nachdem sich auf der naturalistischen Seite viele Geheimnisse der Natur entschlüsseln ließen, bleiben bisher zwei grundsätzliche Fragen unbeantwortet. Dies ist die Frage nach dem Bewusstsein und die noch viel finalere Frage: Wo kommt der Anfang der Welt her? Auch wenn es Wissenschaftler und Philosophen gibt, die meinen, eine solche Frage sei unzulässig, darf eine solche Frage einem Naturwissenschaftler niemals als unzulässig erscheinen. Auch wenn es derzeit noch unmöglich scheint, sie zu beantworten, muss es aus rein logischen Gründen zulässig sein danach zu fragen. Mag sein, dass man auf ein Henne-Ei-Prinzip und damit auf einen Zirkelschluss stößt, der allerdings auch in der Quantenphysik zu finden ist (Goswami u. a. 2018), oder, und das ist der Link zur Glaubensseite, man greift auf die Idee eines erschaffenden Gottes oder göttlichen Wesens zurück. Genau hier treffen sich die beiden Pole zu einer Überlagerungssituation, für die es derzeit keine deterministisch-fixierende Antwort gibt. Allerdings gebietet die Logik, bei Rückgriff auf einen schöpferischen Gott, die unausweichliche Frage: Wo kommt dann dieser Gott her? Ob die Antwort von Löffler die einzig richtige ist (Löffler 2017; in Bergmann 2019), wird wohl weiterhin intensiv diskutiert werden: Er bezieht sich auf Leibnitz, der von einem ewigen Universum ausgeht und es damit begründet, dass ein ,zureichender Grund' für die Existenz dieser Welt, nicht von dieser Welt sein kann. Mit der Quantenphysik drängt sich jedoch die Frage in den Raum: Wie kann es sein, dass eine Interaktion mit/zwischen physikalischen Entitäten, wie sie durch unsere Welt repräsentiert werden, ohne physikalische Verschränkung möglich ist. Denn ein Eingriff auf physikalische Entitäten kann nur physikalischer Natur sein und zieht eine Verschränkung logisch nach sich. Eine Verschränkung, mit der ein schöpferischer Gott automatisch mit dieser Welt verbunden würde und demzufolge nicht mehr als außerhalb stehend definiert werden dürfte.

Mit diesem Gedanken und der offenen Antwort möchte ich diese Arbeit mit Dankbarkeit und Freude abschließen: Dankbar, weil ich die Möglichkeiten hatte, mich dieser Forschung widmen zu dürfen und mit Freude, weil sie mich bei so vielen meiner Fragen, die ich teilweise schon seit der Kindheit mit mir trug, ganz essentiell weitergebracht hat. 
Open Access Dieses Kapitel wird unter der Creative Commons Namensnennung 4.0 International Lizenz (http://creativecommons.org/licenses/by/4.0/deed.de) veröffentlicht, welche die Nutzung, Vervielfältigung, Bearbeitung, Verbreitung und Wiedergabe in jeglichem Medium und Format erlaubt, sofern Sie den/die ursprünglichen Autor(en) und die Quelle ordnungsgemäß nennen, einen Link zur Creative Commons Lizenz beifügen und angeben, ob Änderungen vorgenommen wurden.

Die in diesem Kapitel enthaltenen Bilder und sonstiges Drittmaterial unterliegen ebenfalls der genannten Creative Commons Lizenz, sofern sich aus der Abbildungslegende nichts anderes ergibt. Sofern das betreffende Material nicht unter der genannten Creative Commons Lizenz steht und die betreffende Handlung nicht nach gesetzlichen Vorschriften erlaubt ist, ist für die oben aufgeführten Weiterverwendungen des Materials die Einwilligung des jeweiligen Rechteinhabers einzuholen.

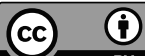

